\title{
Perspective
}

PERSPECTIVE Actualité en histoire de l'art

1 | 2014

L'atelier

\section{De l'atelier au monument et au musée}

From studio to monument to museum

Oskar Bätschmann, Aldo De Poli, Dario Gamboni, Daniel F. Herrmann et Giles Waterfield

Traducteur : Géraldine Bretault

(2) OpenEdition

Journals

Édition électronique

URL : http://journals.openedition.org/perspective/4345

DOI : $10.4000 /$ perspective. 4345

ISSN : 2269-7721

Éditeur

Institut national d'histoire de l'art

Édition imprimée

Date de publication : 1 juin 2014

Pagination : 43-54

ISSN : 1777-7852

Référence électronique

Oskar Bätschmann, Aldo De Poli, Dario Gamboni, Daniel F. Herrmann et Giles Waterfield, « De l'atelier au monument et au musée », Perspective [En ligne], 1 | 2014, mis en ligne le 31 décembre 2015,

consulté le 02 octobre 2020. URL : http://journals.openedition.org/perspective/4345 ; DOI : https:// doi.org/10.4000/perspective. 4345 


\section{De l'atelier au monument et au musée}

Points de vue de Oskar Bätschmann, Aldo De Poli, Daniel F. Herrmann et Giles Waterfield, avec Dario Gamboni

L'évolution récente des musées, avec l'accent mis sur l'événementialité des expositions temporaires et la tendance à la délocalisation par création de filiales, voire de franchises, a donné un relief nouveau aux institutions qui, comme les ateliers d'artistes ayant fait l'objet d'une patrimonialisation, s'inscrivent dans une temporalité plus longue et sont liées pour leur raison d'être à une personne et souvent à un lieu. La conservation des ateliers, comme celle des maisons d'artistes dont ils sont souvent (mais non toujours) une partie, a dépendu d'abord de la piété et servi au culte de l'art et des artistes. Cette dimension cultuelle, particulièrement évidente lorsque les lieux concernés incluent la sépulture de l'artiste, n'est pas incompatible avec la valeur historique ni avec l'intérêt scientifique, lequel doit au contraire en tenir compte et l'intégrer à son propos, mais elle s'oppose au postulat d'autonomie qui a gouverné l'esthétique muséologique moderniste.

Comme l'atelier lui-même, la problématique de la conservation de l'atelier fait partie d'ensembles plus vastes : maisons d'artistes, monuments, lieux de mémoire... Leur spécificité tient au fait qu'ils attirent et retiennent en raison de leur proximité, réelle ou supposée, avec le phénomène de la création artistique. À ce titre, ils appartiennent à la fois au passé dont ils témoignent, et au présent de la réception des œuvres, toujours actualisée par chaque sujet : ils représentent un lieu physique où ces deux moments du temps peuvent entrer en contact.

L'enjeu de cette articulation soulève des questions nombreuses, dont plusieurs sont abordées dans notre débat : celle du cadre de délimitation de l'objet conservé, et du contexte qui lui demeure ou non associé ; celle des conditions de l'authenticité (matérielle, génétique, vécue) de la transmission; celle du rapport entre la dimension mythique de l'atelier, comme représentation et comme attente, et la volonté d'informer objectivement; celle, enfin, de l'actualité de l'atelier au vu de l'évolution des pratiques et des lieux de l'activité artistique. [Dario Gamboni]

Dario Gamboni. Les ateliers, notamment ceux qui ont été conservés, faisaient souvent partie d'ensembles architecturaux et fonctionnels plus vastes, comprenant selon les cas habitation, galerie, entrepôt, etc. Dans quelle mesure est-il légitime de les en isoler? Ce problème se pose-t-il différemment selon l'origine historique et culturelle de l'atelier ?
Oskar Bätschmann, professeur émérite de l'université de Berne et Research Professor à I'Institut suisse pour l'étude de I'art à Zurich, est l'auteur de plusieurs ouvrages, dont The Artist in the Modern World: The Conflict between Market and Self-Expression (1997).

Architecte diplômé et professeur à la faculté d'architecture de l'université de Parme, Aldo De Poli a collaboré à de nombreuses expositions (la Biennale de Venise, Arti \& Architettura...) et a publié sur l'architecture, les maisonsmusées et la muséographie.

Dario Gamboni est professeur d'histoire de l'art à l'université de Genève. Il prépare un ouvrage sur les musées d'artistes et de collectionneurs pour la "Yale Series on the History and Theory of Art Museums » (à paraître).

Daniel F. Herrmann est Eisler Curator \& Head of Curatorial Studies à la Whitechapel Gallery. Conservateur en 2004-2010 à la Scottish National Gallery of Modern Art, il était en charge de la reconstitution de I'atelier d'Eduardo Paolozzi.

Giles Waterfield est directeur des Royal Collection Studies et Associate Lecturer au Courtauld Institute. Il a signé de nombreuses expositions (The Artist's Studio, 2009) et publications académiques (The People's Galleries, à paraître en 2015). 


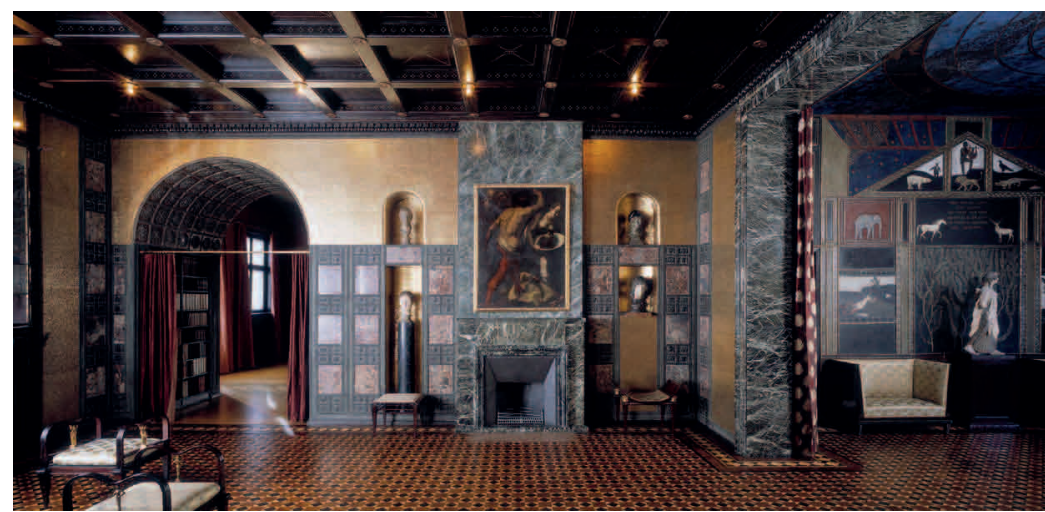

Oskar Bätschmann. Il est sans doute préférable, ou à tout le moins souhaitable, que soient conservés les ensembles architecturaux et fonctionnels dont faisaient partie les ateliers. C'est ce qu'on a heureusement fait avec la Villa Stuck à Munich, par exemple (fig. 1).

Mais le choix concret d'une solution ou d'une autre dépendra toujours moins de la doctrine de conservation à laquelle on adhère, et bien davantage des finances à dis-

1. Le salon et de la salle de musique de Franz von Stuck, construits vers 1897-1898, dans I'actuel Museum Villa Stuck, Munich.
2. Le cabinet de travail de Sigmund Freud, avec le bureau à gauche et le divan à droite, dans l'actuel Freud Museum London.

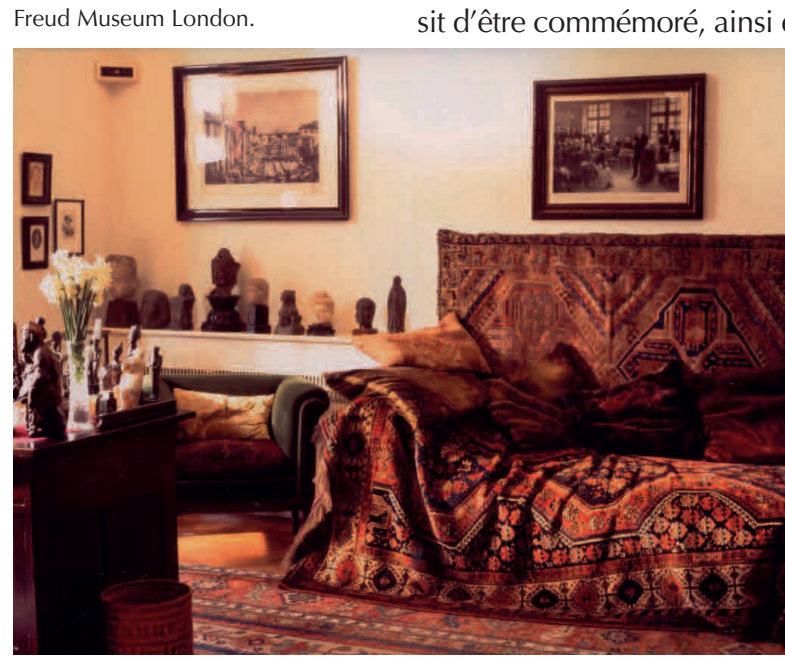
position, de la volonté et de la possibilité qu'une société, une fondation, une ville ou un État assume le soin de veiller à la conservation d'un ensemble. L'expérience commune montre que l'intérêt public pour un atelier d'artiste diminue rapidement une fois que l'attrait de la nouveauté s'est dissipé, et qu'il a tendance à disparaître tout à fait, ce qui a des conséquences graves pour la conservation matérielle.

Giles Waterfield. L'atelier d'artiste, conservé ou en activité, a toujours fonctionné selon une grande variété de modalités : comme le foyer de la créativité artistique individuelle, comme un atelier de production d'œuvres exécutées mais non conçues par des assistants, ou encore comme un lieu d'exposition et de vente d'œuvres d'art, un lieu de vie, une retraite pour la méditation personnelle, un foyer d'hospitalité conviviale ou érotique. L'atelier conservé peut être considéré soit comme une sorte de document, soit comme un lieu de mémoire. Dans le premier cas, il peut être justifié de traiter le studio comme un phénomène isolé, ce qui implique que l'artiste soit placé sous un microscope et analysé comme un individu à part. Dans le second cas - qui, il faut le dire, séduit généralement un large public -, il est impensable d'isoler l'atelier de son environnement, ce qui reviendrait à considérer l'artiste non plus comme un individu, mais comme un simple moteur dans la création des œuvres d'art. La relation entre l'atelier et la demeure ou la galerie, ou toute autre entité ayant subsisté, n'enrichit pas seulement notre compréhension de la personne, elle reflète surtout le processus complexe selon lequel cet artiste (ou ses admirateurs) choisit d'être commémoré, ainsi que le contexte dans lequel son œuvre a évolué. Des notions telles que la « mort de l'artiste » - au sens de mort de l'auteur, de rejet de la biographie - sont, assurément, particulièrement inopérantes dans ce contexte.

L'atelier conservé fait partie d'un mouvement plus large qui vise à conserver, voire à monumentaliser la demeure des hommes (et, moins souvent, des femmes) illustres. Apparu au début du XIX ${ }^{\mathrm{e}}$ siècle, ce mouvement concerne surtout la maison d'écrivain ; il peut aussi s'agir de la demeure d'un compositeur, d'un architecte, d'un homme politique, sans oublier Sigmund Freud (fig. 2). Ces habitations et lieux de travail conservés pourraient être regroupés sous le terme de " musées de personnalité », suggérant non seulement leur association avec tel individu mais aussi le statut particulier que cette personne a acquis au cours de sa vie, voire même après sa mort par le biais du musée. 
Daniel F. Herrmann. Ces dernières années ont vu émerger une tendance à intégrer des environnements d'ateliers au sein des musées d'art. Ces espaces sont pour la plupart des reconstitutions de lieux de travail ayant appartenu à des auteurs uniques. S'ils présentent de grands avantages, ils ne sont pas pour autant exempts d'inconvénients'. Parmi les avantages potentiels, il faut citer l'effet spectaculaire et l'apport didactique $d^{\prime}$ 'une confrontation visuelle directe, la prise en charge immédiate de la conservation du matériel exposé, ainsi qu'un accès élargi pour les chercheurs comme pour les visiteurs ${ }^{2}$. Quant aux écueils éventuels, ils incluent le risque de privilégier la biographie comme principale méthode d'interprétation, et de perpétuer des conventions artistiques traditionnelles au détriment de modes alternatifs de production artistique.

Ces dispositifs muséalisés ne montrent jamais qu'un état unique de l'atelier. Ils suggèrent un locus unicum de production du génie, alors que beaucoup d'artistes disposent de plusieurs lieux où exercer leur activité : planche à dessin, pièce à vivre, atelier $d^{\prime}$ imprimerie, fonderie, etc. Ces présentations mettent en outre l'accent sur un moment particulier et contingent de la production, au lieu de renseigner sur l'évolution des pratiques de l'artiste tout au long de sa vie. Si tel est déjà le cas lorsqu'un atelier d'artiste est converti en musée, l'intégration d'un atelier au sein d'un musée préexistant est encore plus problématique. Dans ce cas, l'espace de production est coupé de son site de développement historique et vidé de sa substance ${ }^{3}$. Coupé de son contexte de production privée, transféré dans un contexte de réception publique et implanté ou greffé sur le corps-hôte du musée, I'atelier devient une transplantation.

Aldo De Poli. Le jugement porté sur I'art contemporain à la fin du XXe siècle contribue à renforcer le processus d'idéalisation de l'artiste et de sa personnalité multiple, présente dans de nombreux domaines. L'œuvre, et bien sûr l'ensemble du microcosme créatif mis en action, devient l'objet d'intérêt et de protection. L'atelier, I'habitation, la bibliothèque, mais également la rue, le quartier, le café, jusqu'au jardin à la campagne et au paysage - en bref, les lieux qui servent de cadre à la vie quotidienne - sont mis en valeur ; ils sont le théâtre des événements culturels, les lieux privilégiés où se déroulent les scènes de la vie, et donc de cette œuvre d'art suprême qu'est l'existence.

Du fait de la large divulgation permise par les moyens de communication de masse, la vie de l'artiste d'aujourd'hui appartient à tous. Cette prise de conscience en vient à conditionner la définition même de la patrimonialisation. L'aspect architectural tant de l'habitation que de l'atelier prend une place prépondérante dans le projet de storytelling de l'ensemble du parcours créatif. Nous ne sommes qu'au début d'un processus de dispersion totale des témoignages historiques, consécutive à I'apparition d'Internet. Après une lente phase de délocalisation des œuvres de l'atelier au musée, l'offre culturelle du futur va connaître une phase inquiétante de dématérialisation du patrimoine.

De nos jours, c'est à travers l'œuvre, réelle et virtuelle, que la vie est racontée ; les lieux de l'artiste sont conservés, non pas dans un souci mal dissimulé de provoquer la surprise, mais bien en tant que preuves documentaires d'une existence. L'artiste est, la plupart du temps, lui-même auteur de l'aménagement des différents espaces de vie, souvent en collaboration avec des architectes et des paysagistes. Mettre en scène des comportements privés, des fragments du quotidien, des objets de peu de prix, voilà la scénographie recherchée pour lui prêter une valeur quasi mythique. 


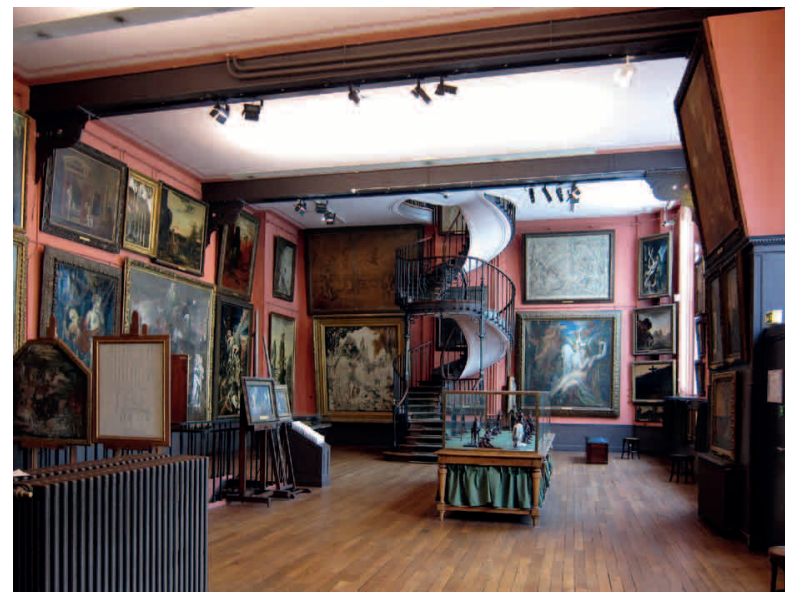

Cette sensibilité nouvelle amène à rendre visibles tous les espaces du travail de création, y compris les espaces intimes et privés. À la permanence des lieux originels vont s'ajouter des centres de documentation offrant des archives et des salles de conférences, des espaces pour l'accueil des visiteurs, et des fondations chargées de faire connaître et de veiller à la sauvegarde d'importantes collections patrimoniales.

Dario Gamboni. Les ateliers qui ont fait l'objet d'une patrimonialisation l'ont été dans des conditions et selon des procédés divers : conservation et/ou reconstitution (partielle ou complète) in situ, déplacement et reconstruction comme entité individuelle ou intégrée à un nouvel ensemble, etc. Certaines conditions et certains procédés vous paraissent-

3. Le grand atelier conçu par Gustave Moreau pour exposer ses œuvres, dans l'actuel Musée national Gustave-Moreau, Paris.

4. L'atelier de sculpture d'Antoine Bourdelle, dans l'actuel Musée Bourdelle, Paris. ils garantir un meilleur résultat, une plus grande authenticité, une plus grande lisibilité ? Avez-vous à l'esprit des réussites et des échecs qui serviraient de modèles respectivement positifs et négatifs?

Giles Waterfield. À mon sens, I'atelier idéal est celui qui a été conservé - ou semble avoir été conservé - en l'état. C'est-à-dire un atelier qui nous permette de pénétrer avec notre imagination dans l'esprit de l'artiste, où les objets contenus dans la pièce ne soient pas seulement des reliques inanimées (comme dans le sanctuaire d'un saint, par une analogie évidente) mais demeurent chargés de sens. Idéalement, l'espace doit parler de lui-même, provoquer une expérience empathique chez le visiteur, en lui permettant d'imaginer l'atelier du temps de son utilisation et de comprendre le processus - technique ou mental - qui aboutissait à la création de l'œuvre d'art. Pour qu'une expérience soit réellement concluante (tout imprégnée soit-elle de préjugés romantiques), le visiteur doit prendre en compte le cadre de l'atelier - s'il était considéré comme un espace privé, et le demeure, ou si son existence est proclamée à l'univers - ainsi que ses abords physiques. Idéalement, il faudrait percevoir la poussière, le froid, l'odeur de la peinture, qui souvent rendaient ces espaces rien moins qu'attrayants. Parmi les ateliers conservés les plus évocateurs et fascinants, il faut citer le Musée Gustave Moreau à Paris (fig. 3). La demeure et I'atelier du grand peintre symboliste furent légués à l'État français, selon sa volonté, en 1897, avec près de 20000 de ses œuvres, dans un geste qui marquait sa foi en son propre génie. Restauré avec soin, son appartement a ouvert au public en 1991. Toujours à Paris, le Musée Bourdelle célèbre la carrière d'un éminent sculpteur, qui vivait et travaillait en ces lieux (fig. 4). À sa mort, son atelier fut conservé par sa famille (une situation fréquente) et des galeries d'exposition lui furent adjointes pour montrer son travail, ainsi que de la sculpture en général. 
L'atelier de Bourdelle forme le cœur du musée. L'atelier d'Antoine Wiertz à Bruxelles (fig. 5) est un cas bien plus complexe, en raison de la perception variable de la qualité artistique de ce peintre d'histoire. La vaste salle d'accueil contient des œuvres - de tradition romantique - qui éblouissaient le public avant sa mort en 1865. Autrefois délabré, le musée Wiertz vient d'être restauré, dans le respect des aspirations de son ancien propriétaire.

L'atelier transporté, puis recréé sur un autre site représente un phénomène fascinant en soi, qu'il s'agisse des fragments de l'atelier de Bertel Thorvaldsen dans son musée éponyme, de l'atelier de Constantin Brancusi à Paris, ou de

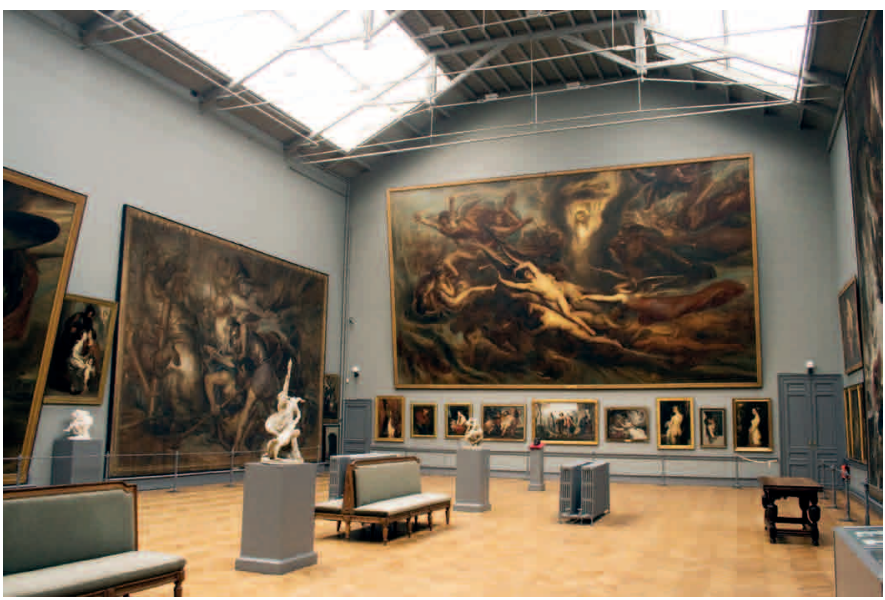
I'atelier de Francis Bacon à Dublin. Que la reconstitution soit une réussite ou un échec, I'atelier ne peut être que radicalement altéré, et ce malgré l'étude la plus fidèle possible de l'original. Il se produit, en fait, une mutation de cet atelier en œuvre d'art de plein droit - une forme de collaboration tacite entre l'artiste d'origine, le conservateur invisible, et même le restaurateur -, une œuvre d'art dans laquelle le visiteur a également un rôle de créateur. Il est (normalement) impossible d'entrer physiquement dans un espace de ce genre, mais on ne peut davantage y entrer par l'imagination; au lieu de cela, il devient soit une puissante œuvre sculpturale (comme dans le cas de Brancusi), soit le spectacle d'un désordre qui titille et mystifie le visiteur (comme à Dublin).

Oskar Bätschmann. Dans le cas de l'atelier Brancusi à Paris, par exemple, on a opté pour une solution de déplacement et pour la reconstruction dans un nouveau contexte. Les espaces qui en résultent, en bordure du Centre Pompidou, doivent être qualifiés de stériles, mais ils demeurent préférables à une destruction pure et simple. La comparaison de l'état actuel de l'atelier Brancusi avec les photographies de l'atelier original démontre que tous les espaces qui ne sont plus habités et qui sont conservés deviennent des espaces morts (fig. 6).

Il y a un devoir primordial, qui doit précéder toute action de modification de l'ensemble d'un atelier et de son contexte, ou de déplacement de telle ou telle partie : celui de procéder à une documentation photographique complète de l'ensemble architectural et fonctionnel. La documentation qui en résulte doit être mise à la libre disposition des chercheurs, et ce autant que possible en ligne. Cette documentation complète, qu'on peut définir comme une conservation immatérielle, est en fait plus importante que la conservation matérielle, qui se révèle souvent fragile.
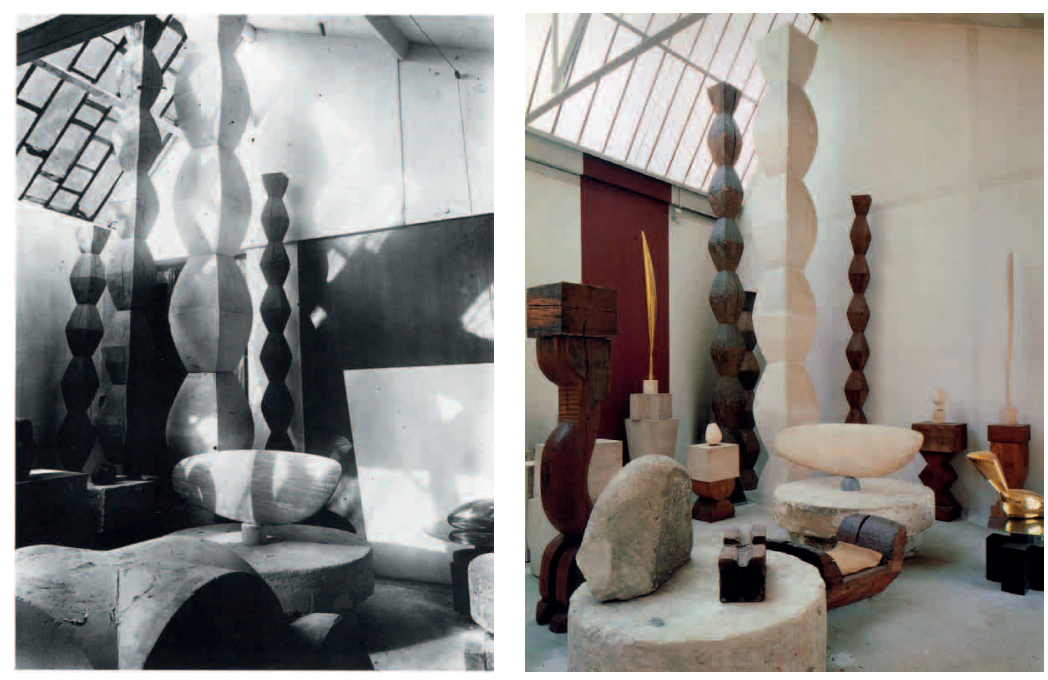

5. Le grand atelier d'Antoine Wiertz, dans l'actuel Musée Wiertz (Musées royaux des Beaux-Arts de Belgique), Bruxelles.

6. L'atelier de Constantin Brancusi : a. photographie de I'artiste montrant I'atelier au 11, impasse Ronsin, vers 1936 ; b. reconstitution de l'atelier au Centre Pompidou, Paris. 
7. Installations architecturales d'Anselm Kiefer dans son domaine La Ribaute, Barjac, vue extraite du film documentaire de Sophie Fiennes, Over Your Cities Grass Will Grow (2010).
Aldo De Poli. Dans I'hypothèse d'une mise en valeur de l'espace d'un atelier, trois possibilités $d^{\prime}$ 'intervention sont envisageables. La première option est celle de la conservation apparente ; la deuxième porte sur l'extension des espaces de vie à l'extérieur du bâtiment, voire sur la construction d'un "paysage d'auteur »; la troisième tente la restitution symbolique et scénographique d'un lieu depuis longtemps disparu.

La première approche, celle de la conservation apparente, étudie les modalités spatiales, les solutions, les arrangements qui doivent être retenus ou expérimentés pour ouvrir au public les espaces intérieurs les plus importants, ceux chargés d'histoire et de mémoire. Dans l'objectif d'une conservation absolue, toutes les transformations nécessaires pour garantir des conditions optimales de fonctionnalité et de sécurité, indispensables pour la mise en valeur de la collection et pour une articulation efficace du parcours de la visite, doivent être assurées. Dès le départ, la capacité expressive du lieu doit faire l'objet d'un examen critique croisé ; des hiérarchies entre les sections sont à déterminer, des valeurs immatérielles sont mises en relation avec des objets physiques, on attribue une forte valeur symbolique à quelques détails traités avec un souci particulier. Au-delà de la rigueur philologique, le parcours de la visite est donc défini dans le but non seulement d'informer et d'éduquer, mais aussi de provoquer la surprise.

La deuxième approche s'intéresse à la construction d'un fragment de " paysage d'auteur ", selon un principe de contrôle du cadre environnemental de l'atelier. Dans ce théâtre de la vie, sont pris en considération des éléments culturels préexistants, qui viennent apporter un complément aux jardins et aux espaces extérieurs proposés. On met en valeur, par exemple, la vue d'une partie de la ville, encadrée par les fenêtres, ou bien, avec un changement de parcours, on cherche à préserver des perceptions sensorielles de la nature. La présence de la ville et de la campagne est alors vue comme une extension inévitable du microcosme des objets et des sentiments personnels. Un fragment de paysage, au même titre qu'un document autographe, peut être considéré comme l'anticipation d'une œuvre d'art. Tel est le cas, par exemple, du domaine La Ribaute, dans le village de Barjac (Gard), un fragment de paysage d'art conçu par Anselm Kiefer qui représente bien plus que le simple atelier de l'artiste (fig. 7). Parfois, la spécificité du lieu ne rend pas légitime d'en

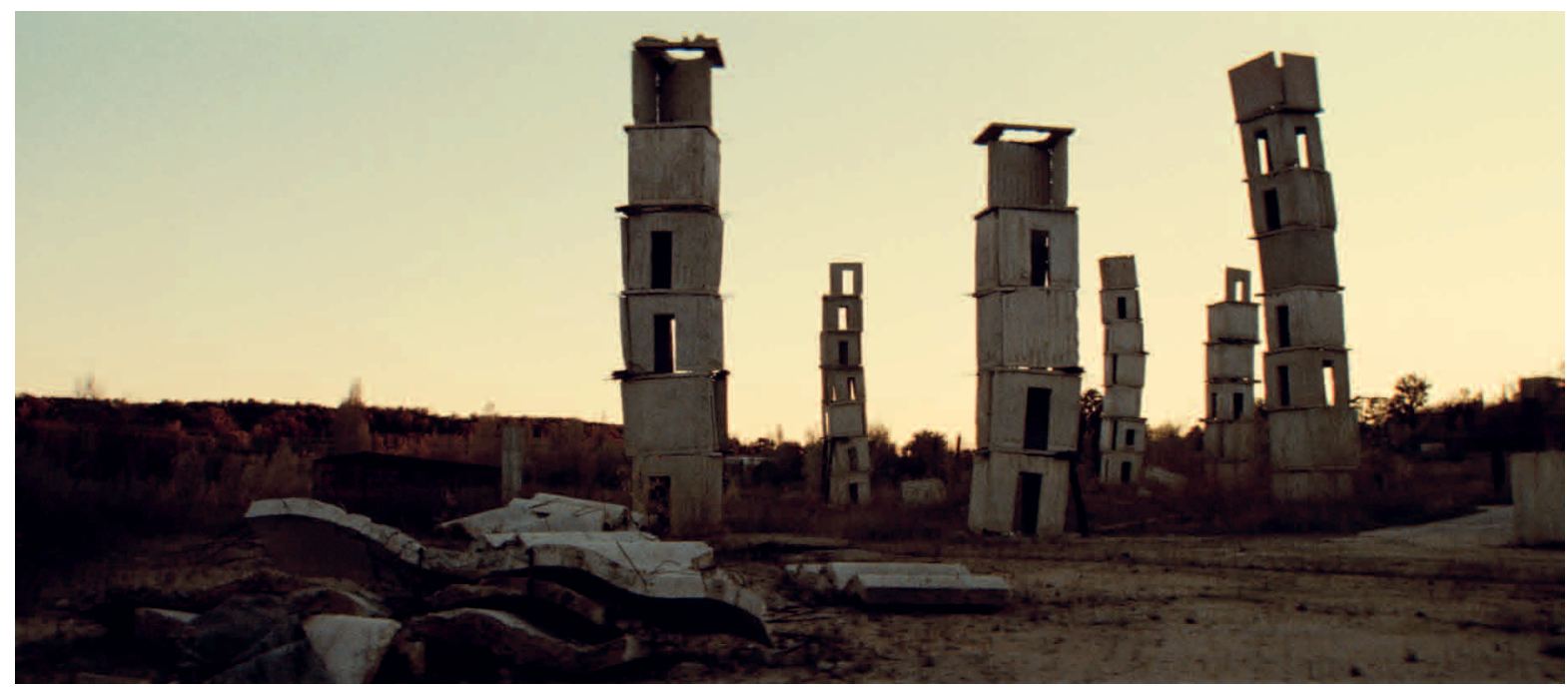


isoler les ateliers. Dans ce cas, on doit élargir la vision rendue publique, jusqu'à doubler voire tripler l'étendue du site. En l'absence d'une campagne d'information adaptée, cependant, cette extension peut ne pas être comprise par ceux qui, par hasard, habitent un tel « écomusée d'art».

Troisième option, on peut privilégier la restitution symbolique d'un site disparu, c'est-à-dire la mise en valeur de lieux de vie qui n'existent plus, qu'ils aient été pillés ou détruits. La valeur sociale qui anime cette nouvelle sensibilité coïncide avec l'attrait de l'inconnu ou la nostalgie. Grâce à des techniques multimédia de simulation et à des reconstructions scénographiques, il a été possible, dans des projets d'aménagement récents, de réaliser des opérations de reconstruction fragmentaire, soit des copies de salles particulières, soit des évocations de sites historiques marquants, aujourd'hui disparus - comme dans l'installation /l sogno di Alberto (2011) de Studio N!03, dédiée à la mémoire des lieux de vie du peintre futuriste Fortunato Depero ${ }^{4}$. Le parcours de la visite, y compris les dépôts documentaires et les reconstructions multimédia, peut s'interrompre et même se fragmenter, avec des ruptures spatiales ou visuelles soudaines créées pour provoquer le dépaysement visuel et narratif. Dans l'installation vidéo Les portes de Cittàdellarte, un travail de Studio Azzurro présenté au Musée du Louvre en 2013 dans le cadre de l'exposition Michelangelo Pistoletto: Année 1, le paradis sur terre, quelques textes écrits, messages ou titres, interviennent dans le parcours. Il ne s'agit plus de notices explicatives, relatives à un objet, mais de voix narratives, de slogans, de témoignages. Totems que l'on peut toucher, installations sonores à écouter, les mots acquièrent de ce fait une épaisseur et une matérialité qui viennent renforcer la perception collective d'un espace désormais partagé.

Daniel F. Herrmann. L'installation d'un atelier d'artiste dans un musée d'art est une anomalie. Traditionnellement, I'atelier d'artiste est un lieu de production. C'est un espace étroitement associé à la personne de l'artiste qui se situe souvent au croisement entre sa vie et son travail. La continuité impliquée entre la vie et la production artistique suggère son authenticité immédiate. À l'inverse, le musée d'art traditionnel est un lieu de réception. En son sein, le contexte original de l'atelier en tant que site de production privée génère un conflit systémique ${ }^{5}$.

Les musées d'art organisent et structurent l'expérience du visiteur à travers des dispositifs de contextualisation ${ }^{6}$. En tant qu'espace de production privé, l'atelier d'artiste ne structure pas l'expérience des visiteurs selon le même mode. Il est dépourvu de panneaux, de cartels, de textes sur les cimaises, de médiateurs et de conventions d'usage public fixées par l'histoire. Alors que les musées guident notre expérience selon une clarté ordonnée, I'iconographie dominante de l'atelier d'artiste au Xx siècle considère le chaos et le désordre comme des indices de créativité. L'implantation d'un atelier d'artiste au sein d'un musée brouille par conséquent les limites traditionnelles du contexte muséal. L'écart entre l'expérience vécue dans chacun de ces deux espaces entretient la confusion : I'atelier transplanté est investi du statut d'œuvre d'art en tant que telle. C'est ce que je constate dans la présentation de l'atelier de Francis Bacon à la Hugh Lane Gallery à Dublin (fig. 8), et c'est un écueil que la reconstitution de l'atelier d'Eduardo Paolozzi à Édimbourg essaie d'éviter constamment (fig. 9).

Dario Gamboni. La fascination exercée par l'atelier tient souvent au mystère qui lui est associé, à l'idée qu'il s'agit d'une sorte de retraite impénétrable où a eu lieu, dans le secret, l'acte créateur. Cette représentation mentale est-elle compatible avec l'accès 

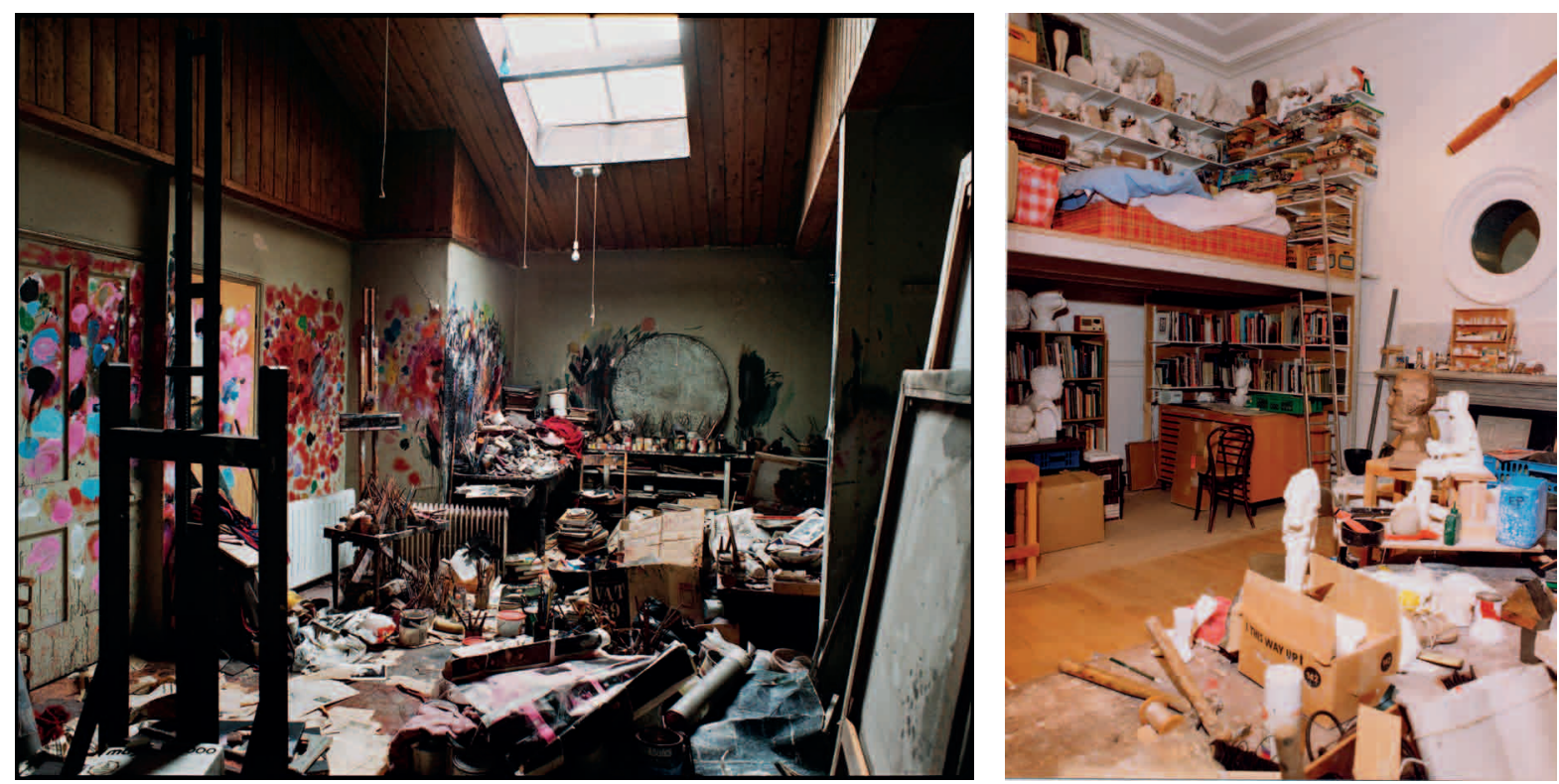

8. L'atelier de Francis Bacon au 7 Reece Mews (Dublin), reconstitution à la Dublin City Gallery The Hugh Lane.

9. L'atelier d'Eduardo Paolozzi, reconstitution à la Scottish National Gallery of Modern Art, Édimbourg. public et collectif qui justifie sa conservation et sa "muséalisation " ? Le modèle de l'atelier ouvert, lieu de représentation et de sociabilité, de collaboration et parfois d'enseignement, s'y prête-t-il mieux? De façon plus générale, peut-on rendre justice à la part de mythe et de culte qui entoure l'atelier d'artiste sans y contribuer soi-même?

Daniel F. Herrmann. Deux problèmes se posent ici à mon sens : premièrement, une grande part de la production artistique est réalisée en situation de collaboration. Or, la plupart des présentations d'ateliers au sein de musées ne rendent pas compte de ces autres contextes de création, des pratiques éditoriales collaboratives, ni de la présence $d^{\prime}$ assistants. Au contraire, ces installations induisent et reposent sur le modèle très traditionnel de l'artiste génial créant dans l'isolement - un modèle dépassé, vivement contesté par les artistes eux-mêmes.

Deuxièmement, je pense qu'il y a un risque de décontextualisation. Lorsqu'il est visité dans son contexte originel, I'atelier d'artiste est à la fois signifiant et significatif de l'authenticité artistique. Cette attente d'authenticité est reportée sur l'atelier reconstitué au sein du musée. En effet, le musée traditionnel repose souvent sur la présence d'un auteur, figuré in absentia par ses œuvres. L'introduction d'un site de production (re)construit dans le cadre muséal entraîne un transfert de ces attentes d'authenticité et d'autorité, de l'œuvre d'art individuelle à l'atelier de l'artiste, et, par extension, de l'œuvre d'art absente à l'artiste absent. Dès lors, la reconstitution d'atelier risque fort de privilégier la biographie par rapport à l'histoire de l'art, et les œuvres d'art courent le danger de devenir de simples reliques.

En vue d'épargner aux ateliers exposés cette atrophie, au regard de l'histoire de l'art, il me semble que les reconstitutions d'ateliers se doivent d'intégrer la cicatrice résultant du processus de greffe. Les institutions doivent mettre en évidence l'écart qui existe entre le contexte de production de la greffe et le contexte de sa réception sur le corps-hôte. Cela peut au moins être accompli par la signalétique, mais doit avant tout se manifester dans des expositions, des présentations et des programmes publics de contextualisation. Quel que soit l'impact visuel de la mise en scène d'un atelier, ce n'est pas sa prétention à l'authenticité, mais bien sa relation au contexte muséologique 
qui confère son importance à ce dispositif. Les meilleures institutions ont une conscience aiguë de ces risques, qu'elles savent même exploiter en faveur de leur programmation artistique : I'exposition de 2006 intitulée The Studio, à la Hugh Lane Gallery, en était un parfait exemple ${ }^{7}$.

Giles Waterfield. Ces ateliers sont des lieux d'une grande puissance évocatrice, très expressifs, qui se suffisent souvent à eux-mêmes. Le conservateur pédagogique peut faire peser une menace décisive sur leur

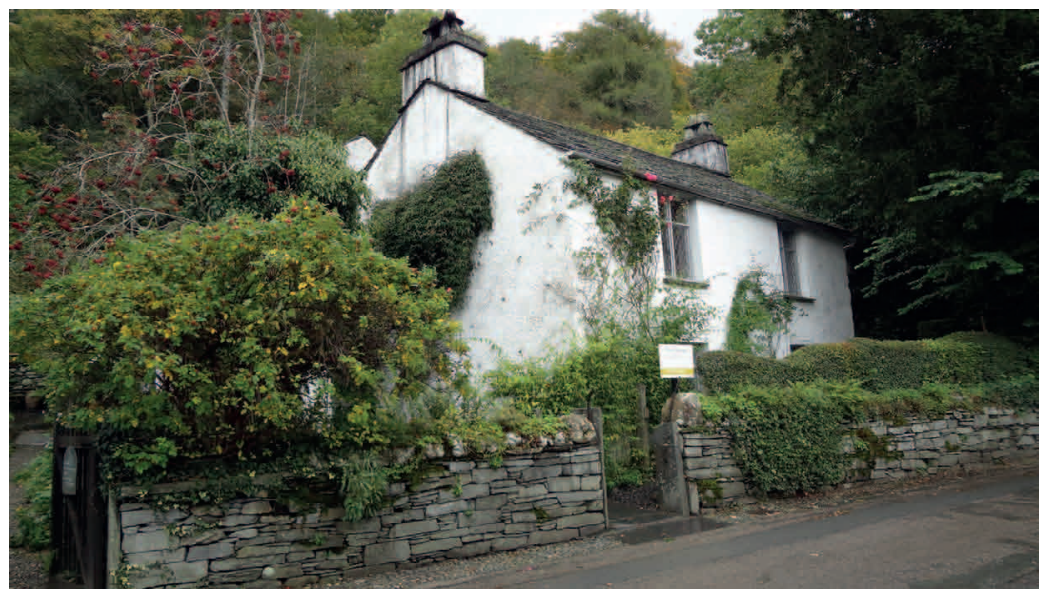
identité, ne pouvant s'empêcher d'interpréter, d'installer des notices et de présenter des vitrines, d'ordonner, d'expliquer. Le Musée Eugène Delacroix en est un parfait exemple : la poésie que dégage I'atelier au cœur de son jardin paisible, creuset de tant de créativité et de tumulte, disparaît derrière une série d'expositions sans pertinence. En Allemagne, une tradition intéressante liée à l'aliénation brechtienne semble être de mise dans les musées consacrés à un personnage illustre. Au Buddenbrookhaus à Lübeck ou à la Beethovenhaus à Bonn, par exemple, toute reconstitution d'un intérieur historique (hormis la présentation d'artefacts associés au héros éponyme) est écartée en faveur d'une quantité de matériel didactique imprimé.

Existe-t-il une raison pour que l'atelier cesse d'être un lieu de sociabilité et d'instruction, un lieu où l'artiste contemporain pourrait puiser son inspiration ? Un bel atelier est un endroit profondément stimulant. Je voudrais citer ici l'exemple d'une maison d'écrivain, Dove Cottage, dans le nord de l'Angleterre, où résidaient William et Dorothy Wordsworth (fig. 10) ; on y accueille régulièrement des séances d'écriture créative pour des publics scolaires et adultes, ce qui semble décupler le pouvoir évocateur de son trésor de souvenirs et d'associations. Le problème consiste à trouver des conservateurs, professeurs ou artistes qui soient capables de traiter ces sites délicats, parfois évanescents, avec toute la sensibilité qu'ils méritent, pour en faire ressortir l'essence même.

Aldo De Poli. À l'origine de toute organisation architecturale se trouve le souhait de disposer d'un agencement formel fixe, considéré comme la condition nécessaire pour développer un certain rite social partagé par la communauté. Dans la longue tradition de l'architecture commencée par Vitruve, une telle intention abstraite de prévoir la forme de l'espace est définie comme utilitas. Dans la préfiguration du projet d'un atelier devenu lieu de mémoire, il faut tout d'abord comprendre la signification ancienne du lieu : le fait d'avoir été un dispositif favorisant l'invention.

Avec l'ouverture au public de l'expérience d'un parcours de visite sur ce même lieu (I'atelier), vient se superposer un nouveau rite. Peut-être plus organisé, mais plus lent, davantage contemplatif et ponctué de pauses. L'ancien atelier est devenu musée. Ces deux réalités, opposées dans leur fonctionnement, ont des racines nombreuses et très profondes dans l'imaginaire collectif.

Comme I'affirme John Serota, le musée d'aujourd'hui doit offrir des contenus différents de ceux de l'enseignement classique et d'Internet. Dans la ville contemporaine, d'autres lieux servent à accueillir la culture. Le consommateur 
culturel d'aujourd'hui est à la recherche d'émotions qu'il veut trouver dans des visites nouvelles de lieux réputés et privilégiés. La condition pour marquer l'actualité de l'œuvre d'art consiste dans la sauvegarde des lieux d'origine de l'artiste, considérés comme la toile de fond indispensable au déroulement de ce rite social partagé. L'avantage symbolique et scientifique de la concentration des témoignages dans un seul lieu permanent tient au fait qu'il renforce la dimension mythique, qu'il s'érige en lieu de mémoire où se croisent les événements collectifs accumulés au cours des années et en des endroits divers.

En dépit de l'importance des racines, il est très fréquent d'assister à la création d'un nouveau musée d'artiste, dans un lieu entièrement différent, qui n'a plus aucun rapport avec le lieu de production original, considéré trop fragile et inadapté à l'accueil des visiteurs. Une politique de conservation des ateliers, protégés autant en raison de leur valeur artistique que pour leur valeur historique, doit prévoir des programmes d'extension dans d'autres lieux, riches d'une spatialité fortement marquée qui s'ajoute au souvenir de l'emplacement originel.

Les mythes remplacent peu à peu la réalité. Les conventions symboliques remplacent la description de la réalité. Le contenu du projet devient de plus en plus complexe; on passe d'un cadre statique à une tension dans le processus et l'interprétation, où le souvenir et la mémoire exigent une redéfinition permanente. On introduit alors de nouveaux contenus dans un programme d'interprétation métaphorique du patrimoine historique, en mettant le sujet dans des conditions susceptibles de traduire l'atmosphère de l'environnement ou l'originalité de l'objet évoqué, en faisant appel à de nouvelles perceptions. De ce nouveau contexte architectural vont naître des émotions, des souvenirs, des perceptions qui se traduisent en expériences, en une synthèse entre réalité et imagination.

Oskar Bätschmann. On ne peut pas éviter de contribuer publiquement, pour les visiteurs, à la mystification du lieu créateur. En revanche, il est possible d'éviter de participer personnellement à la poursuite de ce processus de mystification, contrairement à ce qui a été fait à la maison de Goethe, par exemple, que l'on a conservée et restaurée à Weimar dans le but d'offrir la demeure d'un génie à la vénération.

Dario Gamboni. Le développement de pratiques artistiques comme l'art in situ et la performance, de même que la délégation partielle ou complète de la réalisation matérielle des cuvres, ont amoindri voire aboli le rôle de l'atelier pour nombre d'artistes. D'un autre côté, il arrive fréquemment que les lieux du patrimoine (musées, Kunsthallen et centres d'art, bâtiments historiques, etc.) fonctionnent provisoirement ou durablement comme des lieux de création. Quelles difficultés, quelles limites et quelles nouvelles possibilités ces développements offrent-ils à la patrimonialisation de l'atelier ou de ce qui en tient lieu?

Giles Waterfield. On a beaucoup glosé sur la disparition de l'atelier, sur sa transformation en bureau, sur son manque de pertinence à l'heure actuelle, en raison de la nature de l'art créé aujourd'hui. Pourtant, le studio comme l'atelier restent des lieux puissants pour la création d'œuvres d'art et demeurent indispensables pour de nombreux artistes, que ce soient des lieux de solitude créative ou de sociabilité, ou encore des points de vente pour les œuvres d'art. Une visite dans des ateliers d'artistes à Los Angeles - lieux de monstration et de vente, comme dans le cas de Thomas Houseago (fig. 11) ou Mark Bradford - suggère que la tradition se perpétue avec vigueur, et que ce style ancien de théâtralité indirecte conserve sa prééminence. Je ne vois pas 
en quoi le transfert $d^{\prime}$ une bonne part du travail créatif vers le musée poserait problème. En revanche, l'idée que des œuvres d'art soient créés à destination du musée reste plus délicate. Ce phénomène relativement récent ne manquera pas d'avoir de fortes répercussions sur la nature de I'art en tant que tel, et sur le musée qui I'abrite.

Daniel F. Herrmann. Je $n^{\prime}$ irais pas jusqu'à affirmer que les pratiques contemporaines éliminent le site de production. Il est vrai que certaines formes contemporaines réunissent les sites de production et de réception, mais, contrairement aux présentations muséalisées, elles le

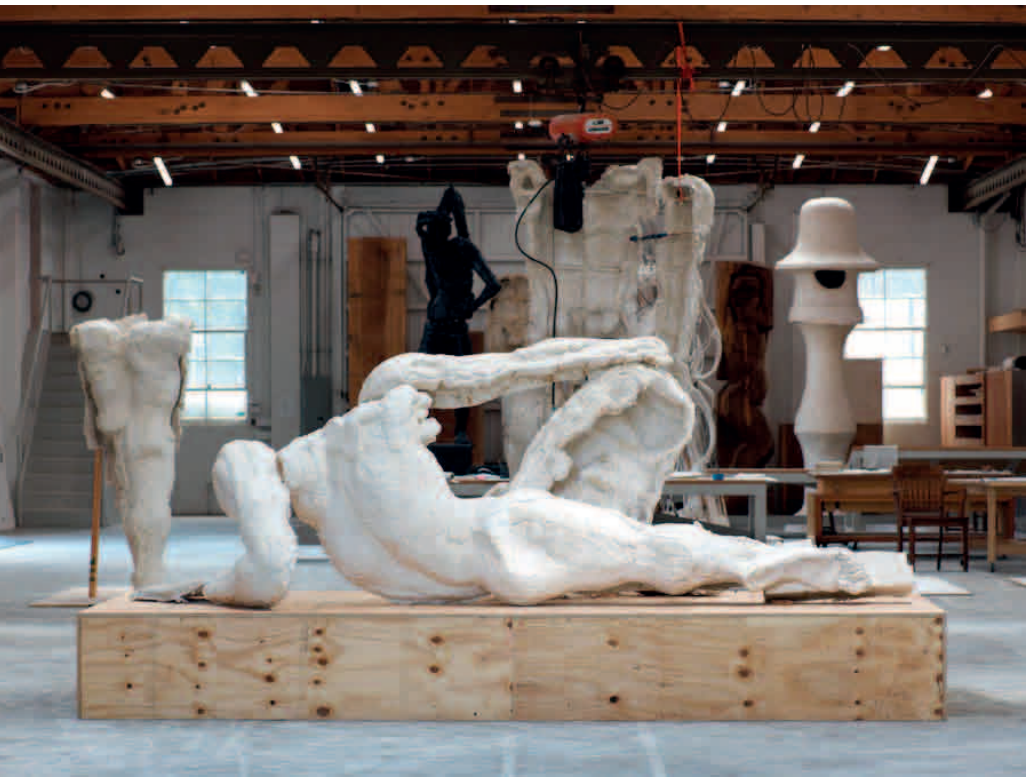
font de manière délibérée et très consciente. Au lieu de chercher à rationaliser après coup la présentation de la production au sein d'un contexte de réception traditionnel, concevoir l'espace muséal comme un lieu de production dès le début est une stratégie pertinente adoptée par un grand nombre de résidences d'artistes, d'expositions et de projets interventionnistes. Ces propositions autorisent une critique institutionnelle ainsi que des expériences formelles, tout en interpellant les artistes, les conservateurs et les visiteurs. Elles s'opposent par principe aux pratiques de présentation statiques, tout en permettant la production de nouvelles œuvres, souvent extérieures au marché de l'art traditionnel. C'est à mon avis une bonne chose.

Pour autant, toute stratégie artistique visant à esthétiser la production court le risque de la nostalgie : je m'intéresse de près aux expérimentations artistiques qui élargissent les définitions de l' « œuvre d'art » pour y inclure le processus de fabrication de manière explicite. Or, si nous muséalisons le «travail » en tant que tel, comment empêcherons-nous sa marchandisation? Et comment empêcheronsnous ces œuvres de n'être que la simple réplique et la représentation des modèles réducteurs de production artistique que nous critiquions auparavant ? Par chance, la fréquentation des artistes contemporains offre de nombreuses occasions d'explorer et d'approfondir ces questions. Ils ont le mérite de mettre en avant des problématiques souvent bien plus intéressantes que celles soulevées par les institutions muséales elles-mêmes.

Oskar Bätschmann. Depuis 1955 environ, les espaces d'exposition sont devenus des lieux qui ont remplacé les ateliers traditionnels, où l'artiste était censé créer quelque chose dans le secret, en espérant que quelque collectionneur, quelque marchand $d^{\prime}$ art ou quelque conservateur de musée s'intéresserait à ses productions. Les lieux d'exposition sont souvent devenus des lieux de création et de réalisation des idées d'un artiste. Il est clair que ces activités artistiques, si elles ne peuvent être conservées, et dans la mesure où elles présentent un intérêt suffisant, doivent elles aussi faire l'objet d'une documentation.
11. L'atelier de Thomas Houseago à Los Angeles, avec, au premier plan, I'œuvre Reclining Figure (For Rome), 2013. 
Aldo De Poli. La vue de tant d'architectures récentes nous amène à faire la réflexion suivante : pour réaliser le plus simple des lieux de mémoire, une culture riche est indispensable. C'est le temps présent qui intéresse, aujourd'hui, un temps où les lieux de création sont partout. De la même façon que les pratiques artistiques peuvent se dérouler dans tant de lieux différents, l'emplacement d'un nouveau musée devient de plus en plus éphémère. Depuis l'arrivée d'Internet, on assiste à un processus de dispersion des témoignages historiques. Avec la dématérialisation du patrimoine, que j'ai déjà évoquée, le nouveau protagoniste de la consommation des biens culturels ne sera plus le groupe social, mais bien l'individu isolé.

La définition formelle de l'institution "musée », au cours des deux derniers siècles, a connu trois phases. De la reconnaissance de la suprématie de la collection, on est passé à une phase centrée sur le public, pour arriver à la phase actuelle, où prévaut la culture du lieu, qui s'incarne dans l'expressivité tranquillisante de la permanence du site urbain et naturel et aussi, dans une moindre mesure, dans l'iconicité dépaysante du bâtiment nouveau.

Cependant, depuis le début du XXI siècle, on voit s'affirmer une quatrième phase : la suprématie de la singularité, des univers privés, des petits mondes sensibles et des lieux de vie chargés de micro-histoires, devenus le dépôt d'une nouvelle anthropologie du temps présent. Le protagoniste est alors l'individu isolé, vu d'abord comme un collectionneur involontaire, puis comme un découvreur curieux de la vie et du travail de l'autre, ou encore comme un inventeur, convaincu du caractère inédit de ses propres parcours dans le musée, enfin comme l'utilisateur inconstant de séquences logiques intermittentes et d'espaces virtuels toujours en mouvement.

Comme on peut le voir parallèlement, dans les projets architecturaux d'autres édifices publics, le musée d'aujourd'hui perd son poids d'histoire, de sobriété et d'efficacité, lequel est remplacé par un charme aimable, une familiarité plaisante, un dépassement des limites spatiales vers l'infini du paysage. Une part de la substance du lieu est véritablement modifiée. Pour reprendre les mots de Hans Magnus Enzensberger, de nos jours, ce ne sont plus des présentations d'objets ou des dépôts du patrimoine matériel qui incarnent le luxe social du $\mathrm{XXI}^{\mathrm{e}}$ siècle, mais le silence et le vide.

Nota bene : ce texte résulte d'échange de courriels.

Les contributions de Daniel F. Herrmann et de Giles Waterfield ont été traduites par Géraldine Bretault.

1. Voir Daniel F. Herrmann, "On Transplants. A Frame Analysis of Artists' Studios in Art Galleries ", dans Jon Wood éd., The Studio in the Gallery: Museum, Reconstruction, Exhibition, Ashgate, 2014 (à paraître) ; Daniel F. Herrmann, Kirstie Meehan, « Lebenserhaltende Massnahmen. Das Paolozzi Studio in Edinburgh », dans Guido Reuter, Martin Schieder éd., Inside/Outside: Das Atelier in der zeitgenössischen Kunst, Petersberg, 2012.

2. Voir Margarita Cappock, Barbara Dawson, Francis Bacon's Studio at the Hugh Lane, Dublin, 2001.

3. Sorcha Carey, "Excavations in the Artist's Studio », dans Apollo, 156/488, 2002, p. 43.
4. L'œuvre a été présentée à l'exposition La magnifica ossessione au Museo di arte moderna e contemporanea di Trento e Rovereto (MART) du 26 octobre 2013 au 16 février 2014.

5. Voir Brian O'Doherty, Studio and Cube: On the Relationship Between Where Art is Made and Where Art is Displayed, Princeton, 2008.

6. Voir Walter Bernhart, Werner Wolf éd., Framing Borders in Literature and Other Media, (Studies in Intermediality, 1), Amsterdam, 2006.

7. The Studio, Jens Hoffman, Christina Kennedy éd., (cat. expo., Dublin City Gallery The Hugh Lane, 2007), Dublin, 2007. 